\title{
Stability Analysis and Power Optimization for Energy Harvesting Cooperative Networks
}

Ioannis Krikidis, Member, IEEE, Themistoklis Charalambous, Member, IEEE, and John S. Thompson, Member, IEEE

\begin{abstract}
In this letter, we investigate the effects of networklayer cooperation in a wireless three-node network with energyharvesting nodes and bursty data traffic. By modelling energy harvesting in each node as a queue (buffer) that stores the received energy, we study the interaction between data and energy queues when only knowledge of the arrival rates is available. The maximum stable throughput (in packets/slot) of the source as well as the required transmitted power for both a non-cooperative and an orthogonal decode-and-forward cooperative schemes are derived in closed-form. We prove that cooperation achieves a higher maximum stable throughout than direct link for scenarios with poor energy arrival rates.
\end{abstract}

Index Terms-Cooperative networks, energy harvesting, power optimization, stable throughput.

\section{INTRODUCTION}

I $\mathrm{N}$ traditional network architectures, the nodes are characterized by limited energy resources and efficient protocols are needed to maximise lifetime or energy savings [1]. On the contrary, energy harvesting or energy scavenging techniques enable wireless nodes to be continuously recharged by the surrounding environment. An energy harvester extracts energy from nature (via solar cells, the piezoelectric effect, vibration, etc.) [2] and converts it to electrical energy. This advance introduces a new vision for wireless networks which necessitates the development of new models that capture the energy harvesting capability, as well as new protocols and techniques that efficiently exploit the harvested energy.

Recent studies reexamined existing transmission techniques and adapted/optimized them in order to take into account the arrivals of the harvested energy. In [3] the authors propose a rate adaptation technique that minimizes the transmission completion time according to the traffic load and the energy harvesting profile by assuming that the energy harvesting times and amounts are known before transmission; this idea is generalized in [4] for a broadcast scenario. Work in [5] models the energy harvesting aspect as an energy buffer and analyzes an adaptive power optimization that minimizes the mean delay in the data queue by simultaneously ensuring stability. Based on a similar energy harvesting model, the authors in [6] investigate

Manuscript received September 26, 2011; revised November 03, 2011; accepted November 04, 2011. Date of publication November 08, 2011; date of current version November 21, 2011. The associate editor coordinating the review of this manuscript and approving it for publication was Prof. Min Dong.

I. Krikidis and T. Charalambous are with the Department of Electrical and Computer Engineering, Faculty of Engineering, University of Cyprus, Nicosia, Cyprus 1678 (e-mail: krikidis@ucy.ac.cy; themis@ucy.ac.cy).

J. S. Thompson is with the Institute for Digital Communications, University of Edinburgh, Edinburgh EH9 3JL, U.K. (E-mail: john.thompson@ed.ac.uk).

Color versions of one or more of the figures in this paper are available online at http://ieeexplore.ieee.org.

Digital Object Identifier 10.1109/LSP.2011.2175382 joint optimization of an (adaptive) scheduling and power control problem for scenarios with multiple users. In addition, the optimal use of the harvested energy for different energy profiles and storage capabilities is discussed in [7] using the outage probability as a performance metric. The impact of the energy harvesting capability on the stability region of a cognitive radio system with bursty traffic is analyzed in [8]; in that work the authors assume a statistical knowledge of the energy arrivals and study energy harvesting at the network protocol level. On the other hand, the design of new protocols for cooperative networks with energy harvesting nodes seems to be a promising research direction that incorporates cooperative benefits (diversity, capacity, etc.) with the energy harvesting concept. The impact of energy harvesting on an Amplify-and-Forward cooperative scheme with relay selection is analyzed in [9] in terms of symbol error probability by using a probabilistic energy model; while a scheduling policy for Decode-and-Forward (DF) cooperative networks with energy harvesting node is investigated in [10] for a simple energy harvesting Markovian model. However, the impact of the energy harvesting at the network layer (cooperation implemented in a packet level, i.e., [11], [12] and references therein) has not yet been reported in the open literature. In this paper, we will adopt a packet-based network view of cooperation and harvested energy with bursty arrivals.

In this paper, we study energy harvesting for a simple three-node network topology (source-relay-destination) without channel-state information (CSI) at the transmitters and when only a statistical knowledge of the packets and energy arrivals is available. The interplay between bursty data traffic and energy harvesting is analyzed at the network layer and the maximum stable throughput is analyzed for both a non-cooperative protocol and an orthogonal DF cooperative scheme. The optimal transmitted power that achieves the maximum stable throughput is also derived for both considered scenarios and for different energy profiles. We show that orthogonal cooperation outperforms direct transmission for poor energy arrivals rates while the direct link seems to be a better solution for scenarios with high energy arrival rates. To the best of our knowledge network-layer cooperation for a wireless network with energy harvesting nodes and a statistical knowledge of the packets and energy arrivals rates is reported in this paper for the first time.

\section{SYSTEM MODEL}

We assume a simple network topology consisting of one source $S$, one relay node $R$ (without own traffic and is used for the case of cooperation) and one destination $D$; the nodes $S$, $R$ are energy harvesting devices that extract energy from the surrounding environment. All nodes are half-duplex and thus they cannot transmit and receive simultaneously. For the sake of simplicity, a normalized linear geometry is assumed, with the distance between the source and the relay equal to $0<\delta<1$ and the distance between the relay and the destination equal to $1-\delta$. The source node transmits with power $P_{S}$ and the 
relay node with power $P_{R}$ (transmitted power for one packet), and path-loss attenuation is taken into account by assuming received power decreases proportional to $d_{i, j}^{-\beta}$ where $d_{i, j}$ is the Euclidean distance between transmitter $i$ and receiver $j$ and $\beta(2 \leq \beta \leq 4)$ is the path-loss exponent. Again, for the sake of simplicity, we assume that the energy consumed in a node concerns the transmission process and therefore, processing or maintenance energy cost is considered to be negligible. However, the model parameters can easily be adjusted to account for such energy costs.

Each of the source and the relay nodes is equipped with two queues (buffers) of infinite capacity ${ }^{1}$ to store data packets and harvested energy. More specifically, the source node holds the queues $Q_{S}$ and $Q_{\epsilon}$ to store its own data packets and the received harvested energy, respectively, while the relay node holds the queue $Q_{S R}$ to store packets relayed for $S$ and $Q_{\epsilon^{\prime}}$ to store its own harvested energy. With this network model, we will investigate the system performance in terms of stable throughput ${ }^{2}$. In order to model bursty arrivals for both the data and the energy queues, data packets and received energy (packets) are generated at the nodes $S, R$ according to stationary Poisson processes with rates $\lambda_{S}$ (in packets/slot) for the data queue $Q_{S}$, and $\lambda_{\epsilon}, \lambda_{\epsilon^{\prime}}$ (in energy units/slot) for the energy queues $Q_{\epsilon}$ and $Q_{\epsilon^{\prime}}$, respectively. Time is assumed to be slotted, such that the transmission time of a packet is equal to one time slot; due to this time normalization the numerical values of energy and power are identical in one time slot and therefore both terms are used interchangeably throughout the paper.

All wireless links exhibit fading and additive white Gaussian noise (AWGN). The fading is assumed to be stationary, with frequency non-selective Rayleigh block fading. This means that the fading coefficients $h_{i, j}$ (for the $i \rightarrow j$ link) remain constant during one slot, but change independently from one slot to another according to a circularly symmetric complex Gaussian distribution with zero mean and variance $\sigma_{i, j}^{2}=d_{i, j}^{-\beta}$ for the link $i \rightarrow j$; also we denote $\rho_{i, j} \triangleq 1 / \sigma_{i, j}^{2}$. Furthermore, the variance of the AWGN is assumed to be normalized with zero mean and unit variance. Each data packet contains $R_{0}$ bits which corresponds to a spectral efficiency of $R_{0}$ bits per channel use (BPCU) and each link $i \rightarrow j$ is characterized by the success probability $\overline{\mathbb{P}}_{i, j}\left(P_{i}\right) \triangleq \mathbb{P}\left\{\log \left(1+P_{i}\left|h_{i, j}\right|^{2}\right)\right.$ $\left.>R_{0}\right\}=\exp \left(-\rho_{i, j}\left(2^{R_{0}}-1\right) / P_{i}\right)$, which denotes the probability that the link $i \rightarrow j$ is not in outage. An outage occurs when the instantaneous capacity of the link $i \rightarrow j$ is lower than the transmitted spectral efficiency rate $R_{0}$. The channel is assumed to be known only at the receivers (but not at the transmitters). Furthermore, the retransmission process is based on an Acknowledgement/Negative-Acknowledgement (ACK/NACK) mechanism, in which short-length error-free packets are broadcasted by the destinations (e.g., destination node or relay node) over a separate narrow-band channel in order to inform the network about the packets' reception status.

\section{Stability ANALYSIS AND OPTIMAL POWER ALLOCATION POLICY}

In this section, we determine the maximum stable throughput and the related power allocation policy for the non-cooperative

\footnotetext{
${ }^{1}$ If in practice these buffers are large enough compared to packet size and energy consumed in a slot, this is a reasonable approximation [5].

${ }^{2}$ It is defined as the set of data arrival rates for which the data queues in the system are stable. If $Q_{i}^{t}$ is the length of the queue $Q_{i}$ at the beginning of time slot $t$, the queue is stable if $\lim _{x \rightarrow \infty} \lim _{t \rightarrow \infty} \mathbb{P}\left\{Q_{i}^{t}<x\right\}=1$.
}

protocol and the DF orthogonal cooperative scheme. Given that all system parameters are stationary (arrival and service processes, channel gains), the stability of the data queues is determined from Loynes' Theorem [13].

\section{A. No Cooperation}

In the non-cooperative protocol, the source node transmits its data without any assistance; this case is used as a baseline scenario in order to evaluate the benefits of the cooperative scheme. In each time slot, when node $S$ transmits a packet, if the destination $D$ decodes the packet successfully, it sends back an ACK and the packet exits the system; otherwise, if $D$ does not decode the packet, the packet will remain at $S$ 's queue for retransmission. In order to decouple the interaction between the data queue and the energy queue and be able to characterize the stability region of the system, we assume a stochastic dominant system where the source node transmits dummy packets and thus continuously consumes power $P_{S}$ even for the case that it becomes empty (a similar method is used in [8] in order to characterize stability). If $P_{S}$ denotes the energy/power consumed at the source node for the transmission of a data packet, the stability of the data queue $Q_{S}$ requires

$$
\begin{aligned}
\lambda_{S}<\mu_{S}\left(P_{S}\right) & =\overline{\mathbb{P}}_{S, D}\left(P_{S}\right) \mathbb{P}\left(P_{S}\right) \\
& =\exp \left(-\frac{\rho_{S, D}\left(2^{R_{0}}-1\right)}{P_{S}}\right) \mathbb{P}\left(P_{S}\right)
\end{aligned}
$$

where $\mu_{i}$ denotes the service rate for the queue $i$ and $\mathbb{P}\left(P_{S}\right)$ denotes the probability that the energy/power $P_{S}$ is available at the energy queue $Q_{\epsilon}$ and is given as

$$
\mathbb{P}\left(P_{S}\right)= \begin{cases}\frac{\lambda_{\epsilon}}{P_{S}}, & \text { if } P_{S}>\lambda_{\epsilon}, \\ 1, & \text { otherwise. }\end{cases}
$$

The above stability conditions are sufficient and necessary conditions for the stability of the original system [8]. Based on the construction of the dominant system, the data queues of the dominant system are always larger in size that those of the original system, provided the queues start with identical initial conditions in both systems. Therefore, If for some $\lambda_{S}$ the queue $Q_{S}$ is stable in the dominant system then it must be stable also in the original system (e.g., the source can transmit in a higher power $\left.P_{S}\right)$. Conversely, if for some $\lambda_{S}$ in the dominant system, the queue $Q_{S}$ saturates, then it will not transmit dummy packets and thus the behavior of the dominant system becomes identical to that of the original system. Therefore, the original system and the dominant system are indistinguishable at the boundary points and thus have the same stability region.

Given that the maximum departure rate depends on the transmitted power, the maximum stable throughout is given by solving the following optimization problem:

$$
\begin{aligned}
\max _{P_{S}} & \mu_{S}\left(P_{S}\right) \\
& = \begin{cases}\max _{P_{S}} \exp \left(-\frac{\rho_{S, D}\left(2^{R_{0}}-1\right)}{P_{S}}\right) \frac{\lambda_{\epsilon}}{P_{S}}, & \text { if } P_{S}>\lambda_{\epsilon}, \\
\max _{P_{S}} \exp \left(-\frac{\rho_{S, D}\left(2^{R_{0}}-1\right)}{P_{S}}\right), & \text { elsewhere. }\end{cases} \\
& = \begin{cases}\exp (-1) \frac{\lambda_{\epsilon}}{\rho_{S, D}\left(2^{R_{0}}-1\right)}, & \text { with } P_{S}^{*}=\rho_{S, D}\left(2^{R_{0}} 1\right)>\lambda_{\epsilon}, \\
\exp \left(-\frac{\rho_{S, D}\left(2^{R_{0}}-1\right)}{\lambda_{\epsilon}}\right), & \text { with } P_{S}^{*}=\lambda_{\epsilon} .\end{cases}
\end{aligned}
$$

The above result shows that for the case of a poor energy profile (i.e., when $\lambda_{\epsilon}<\rho_{S, D}\left(2^{R_{0}}-1\right)$ ) the source transmits 
with a power $P_{S}^{*}=\rho_{S, D}\left(2^{R_{0}}-1\right)$ that is higher than the energy arrival rate; for all other cases a transmitted power/energy equal to the arrival energy rate maximizes the stable throughout.

\section{B. Orthogonal DF Cooperation}

In this case, a relay node assists the source to deliver its packets to the destination using two time slots. The adopted cooperative protocol is a "network" version of the orthogonal DF cooperative scheme where relay assistance is performed in an orthogonal channel. Since our main target is to study the interaction between the data and energy queues for a cooperative network topology, the orthogonal DF scheme is a illustrative case study and a useful guideline for more advanced cooperative schemes. More specifically, we assume that time slots are assigned to the source and the relay node according to a Time-Division Multiple Access (TDMA) policy; odd slots are dedicated for source transmission and even slots for relay transmission. The cooperation strategy is stated as follows: (1) during odd time slots, node $S$ transmits a packet, if the destination $D$ decodes the packet successfully, it sends back an ACK and the packet exits the system; (2) otherwise, if $D$ does not decode the packet but $R$ decodes the packet, $R$ sends back an ACK and keeps the packet in its queue for retransmission, upon receiving the ACK from $R, S$ will drop the packet (the relay node observes the ACK/NACK signal transmitted by the destination, and hence it knows whether it is the only node that decodes the source signal [12]); (3) if neither $R$ nor $D$ decode the packet, the packet will remain at $S$ 's queue for retransmission; (4) during even time slots, node $R$ relays source packets (which are stored at $\left.Q_{S R}\right)$ via the link $R \rightarrow D$; if this packet is decoded at the destination, it is removed from $Q_{S R}$ queue; otherwise, it remains in $Q_{S R}$ for future retransmission. In order to determine the stable throughput for this case, we need to study stability conditions for both data queues $Q_{S}$ and $Q_{S R}$. It is worth noting that in order to characterize the stability of the system, we assume a dominant system where the nodes (source or relay) transmit dummy packets when their queues becomes empty. By using the same arguments presented in Section III-A, the constructed dominant system ensures the stability of the original system while it becomes indistinguishable with the original system for the saturation case.

1) Stability Conditions at the Source: As in the non-cooperative case, the source $S$ establishes stability for the data queue if the rate by which data are transmitted to the destination $D$ $\left(\mu_{S, D}\right)$ is higher than the data rate $\left(\lambda_{S}\right)$ coming into the buffer. A packet is removed from the source queue $Q_{S}$ when the time slot is assigned to the source node, and (a) the packet is decoded at the destination, or (b) the packet is decoded at the relay node. Hence, the condition is as follows:

$$
\begin{aligned}
\lambda_{S}<\mu_{S}\left(P_{S}\right)= & \frac{1}{2}\left[\overline{\mathbb{P}}_{S, D}\left(P_{S}\right)\right. \\
& \left.+\left(1-\overline{\mathbb{P}}_{S, D}\left(P_{S}\right)\right) \overline{\mathbb{P}}_{S, R}\left(P_{S}\right)\right] \mathbb{P}\left(P_{S}\right)
\end{aligned}
$$

where the factor $1 / 2$ correponds to the TDMA policy using two time slots and $\mathbb{P}\left(P_{S}\right)$ is given by

$$
\mathbb{P}\left(P_{S}\right)= \begin{cases}\frac{2 \lambda_{\epsilon}}{P_{S}}, & \text { if } P_{S}>2 \lambda_{\epsilon} \\ 1, & \text { otherwise. }\end{cases}
$$

2) Stability Conditions at the Relay: Likewise, at the relay $R$, it is required that the rate $\left(\mu_{S R}\right)$ by which data are transmitted to the destination $D$ is higher than the data rate $\left(\lambda_{S R}\right)$ coming into the buffer by the transmitter $S$, i.e., $\lambda_{S R}<\mu_{S R}$. Hence, the stability condition can be expressed as follows [12]:

$$
\frac{1}{2} \frac{\lambda_{S}}{\mu_{S}\left(P_{S}\right)}\left(1-\overline{\mathbb{P}}_{S, D}\left(P_{S}\right)\right) \overline{\mathbb{P}}_{S, R}\left(P_{S}\right) \mathbb{P}\left(P_{S}\right)<\frac{1}{2} \overline{\mathbb{P}}_{R, D}\left(P_{R}\right) \mathbb{P}\left(P_{R}\right) \text {. }
$$

Therefore

$$
\begin{aligned}
\lambda_{S} & <\mu_{S}\left(P_{S}\right) \frac{\overline{\mathbb{P}}_{R, D}\left(P_{R}\right) \mathbb{P}\left(P_{R}\right)}{\left(1-\overline{\mathbb{P}}_{S, D}\left(P_{S}\right)\right) \overline{\mathbb{P}}_{S, R}\left(P_{S}\right) \mathbb{P}\left(P_{S}\right)} \\
& <\mu_{S}\left(P_{S}\right) \frac{\max _{P_{R}}\left[\overline{\mathbb{P}}_{R, D}\left(P_{R}\right) \mathbb{P}\left(P_{R}\right)\right]}{\left(1-\overline{\mathbb{P}}_{S, D}\left(P_{S}\right)\right) \overline{\mathbb{P}}_{S, R}\left(P_{S}\right) \mathbb{P}\left(P_{S}\right)} \\
& =\mu_{S}\left(P_{S}\right) \frac{\Psi\left(P_{R}\right)}{\left(1-\overline{\mathbb{P}}_{S, D}\left(P_{S}\right)\right) \overline{\mathbb{P}}_{S, R}\left(P_{S}\right) \mathbb{P}\left(P_{S}\right)}
\end{aligned}
$$

where $\mathbb{P}\left(P_{R}\right)$ again denotes the required probability of relay transmission to ensure stability of the energy input/output queue at the relay:

$$
\mathbb{P}\left(P_{R}\right)= \begin{cases}\frac{2 \lambda_{\epsilon^{\prime}}}{P_{R}}, & \text { if } P_{R}>2 \lambda_{\epsilon} \\ 1, & \text { otherwise. }\end{cases}
$$

(the factor 2 in the above expression is related to the TDMA policy) and

$$
\begin{aligned}
& \Psi\left(P_{R}\right)= \\
& \begin{cases}\exp (-1) \frac{2 \lambda_{\epsilon^{\prime}}}{\rho_{R, D}\left(2^{R_{0}}-1\right)}, & P_{R}^{*}=\rho_{R, D}\left(2^{R_{0}}-1\right)>2 \lambda_{\epsilon^{\prime}}, \\
\exp \left(-\frac{\rho_{R, D}\left(2^{R_{0}}-1\right)}{2 \lambda_{\epsilon^{\prime}}}\right), & \text { elsewhere with } P_{R}^{*}=2 \lambda_{\epsilon} .\end{cases}
\end{aligned}
$$

Note that the TDMA policy decouples the interaction between source and relaying queues and therefore the maximization of the service rate for the relaying queue is independent of the source queue. The total maximum stable throughput for the source stems from the maximum throughput that can be established by the intersection of the stability regions for both the source and the relay nodes [12, Sec. IV-B]. By combining (4) and (6), the total maximum stable throughput is given by the following optimization problem:

$$
\begin{aligned}
\lambda_{S} & <\max _{P_{S}} \min \\
& {\left[\mu_{S}\left(P_{S}\right), \mu_{S}\left(P_{S}\right) \frac{\Psi\left(P_{R}\right)}{\left(1-\overline{\mathbb{P}}_{S, D}\left(P_{S}\right)\right) \overline{\mathbb{P}}_{S, R}\left(P_{S}\right) \mathbb{P}\left(P_{S}\right)}\right] }
\end{aligned}
$$

which yields the optimal transmitted power for the source node equal to

$$
P_{S}^{*}=\arg \max _{P_{S}} \min \left[\frac{1}{\alpha}, \frac{\Psi\left(P_{R}\right)}{\left(1-\overline{\mathbb{P}}_{S, D}\left(P_{S}\right)\right) \overline{\mathbb{P}}_{S, R}\left(P_{S}\right)}\right]
$$

with

$$
\alpha= \begin{cases}1, & \text { if } P_{S} \leq 2 \lambda_{\epsilon} \\ P_{S}, & \text { if } P_{S}>2 \lambda_{\epsilon}\end{cases}
$$

\section{NUMERICAL RESULTS}

Computer simulations were carried out in order to evaluate the performance of the proposed schemes. The simulation set-up follows the system model of Section II with $\delta=0.5, \beta=2$, 


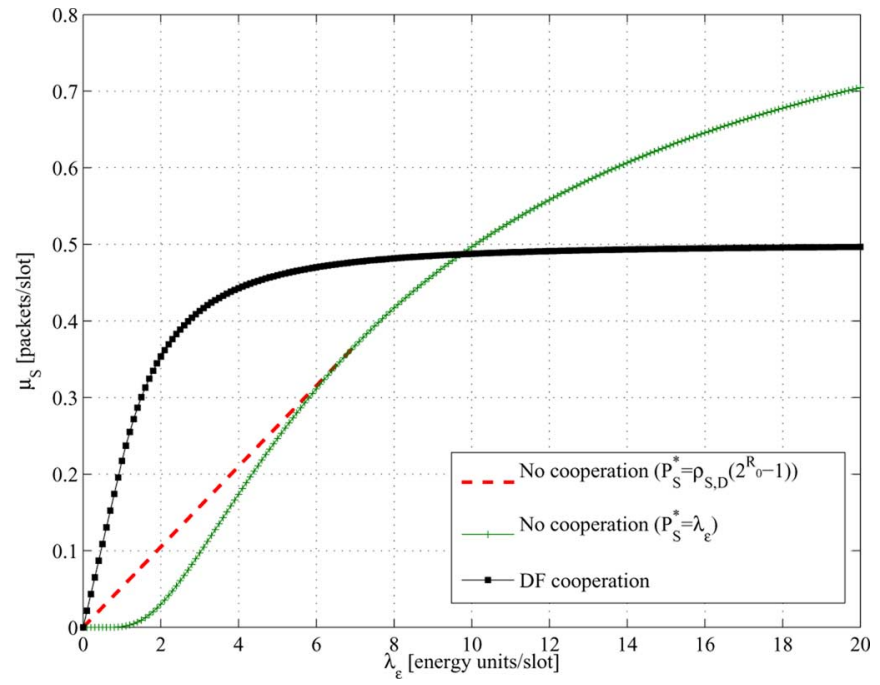

Fig. 1. Stable throughput versus $\lambda_{\epsilon}$ for no cooperation and cooperation; $\delta=$ $0.5, \beta=2, R_{0}=3 \mathrm{BPCU}$ and $\lambda_{\epsilon}{ }^{\prime}=\lambda_{\epsilon}$.

$R_{0}=3 \mathrm{BPCU}$ and $\lambda_{\epsilon}=\lambda_{\epsilon^{\prime}}$. In Fig. 1 we plot the maximum achievable stable throughput $\left(\lambda_{S}\right)$ versus the energy arrival rate for the considered non-cooperative and DF cooperative schemes. The main observation is that the cooperative protocol achieves a higher stable throughput than the direct link for scenarios with a poor energy profile $\left(\lambda_{\epsilon}<10\right.$ energy units/ packet). For these scenarios, which are characterized by a critical energy constraint, cooperation and the related short-range transmissions ensure a more efficient use of the harvested energy and provide a higher maximum stable throughput, despite the bottleneck of the orthogonal transmission. However, the cooperative scheme due to the TDMA limitation converges to a maximum stable throughout equal to $1 / 2$ as the energy arrival rate increases. On the other hand, for high energy arrival rates, the source node has enough energy in order to deliver its data to the destination without suffering from the TDMA limitation and therefore outperforms the cooperative scheme. As for the direct link, we can see that for the low energy arrival rates, the transmission policy $P_{S}^{*}=\rho_{S, D}\left(2^{R_{0}}-1\right)>\lambda_{\epsilon}$ achieves a higher stable throughput than the policy $P_{S}^{*}=\lambda_{\epsilon}$; this observation validates our analysis in (3). Finally, Fig. 2 depicts the power transmitted by the source node under the DF cooperative scheme. We can see that the optimal transmission policy for energy arrival rates with $\lambda_{\epsilon}>1$ is $P_{S}^{*}=2 \lambda_{\epsilon}$ which is found by solving the optimization problem in (10).

\section{CONCLUSION}

This letter has dealt with the impact of the energy harvesting capability on a simple wireless network with orthogonal DF cooperation, when a statistical knowledge of the data and energy arrival rates is available. We have explored the interplay between energy and data queues and characterized the maximum stable throughput and the related power allocation policy for the case with and without cooperation. We have proved that network-layer orthogonal cooperation uses the harvested energy more efficiently for scenarios with a poor energy profile, while direct link is a suitable solution for high energy arrival rates. This work demonstrates the benefits of an energy harvesting

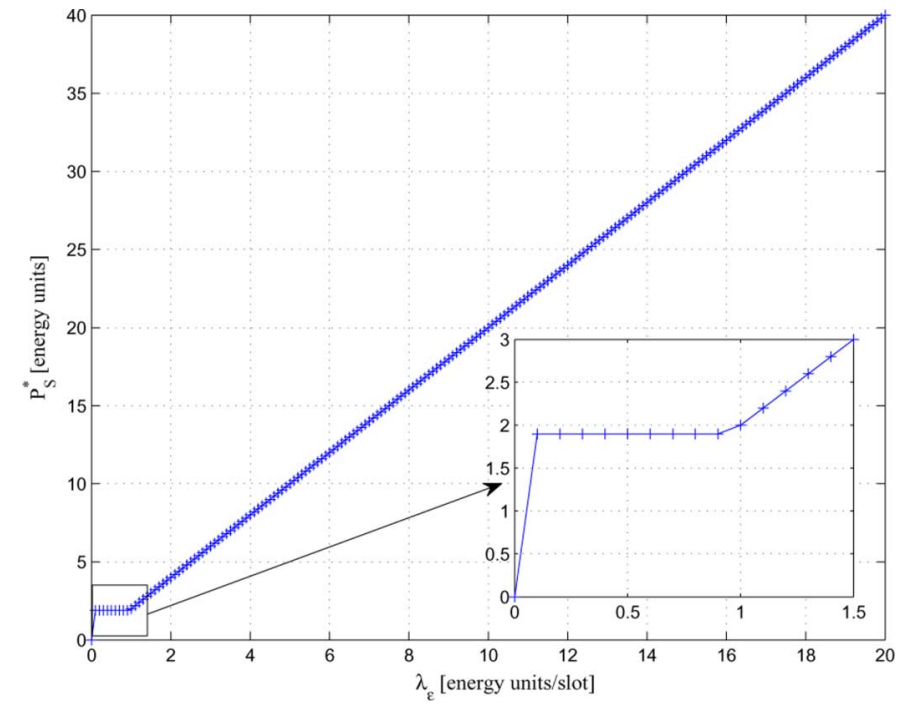

Fig. 2. Optimal source transmitted power $\left(P_{S}^{*}\right)$ versus $\lambda_{\epsilon}$ for cooperation; $\delta=$ $0.5, \beta=2, R_{0}=3 \mathrm{BPCU}$ and $\lambda_{\epsilon^{\prime}}=\lambda_{\epsilon}$.

cooperative system and constitutes a useful guideline for more complex network topologies (multiple nodes) and more sophisticated cooperative protocols.

\section{REFERENCES}

[1] C. Han et al., "Green radio: Radio techniques to enable energy efficient wireless networks," IEEE Commun. Mag. (Special Issue: Green Communications), vol. 49, pp. 46-54, Jun. 2011.

[2] K. K. Win, X. Wu, W. J. Wen, R. Kumar, and S. K. Panda, "Efficient solar energy harvester for wireless sensor nodes," in Proc. IEEE Int. Conf. Commun. Systems, Singapore, Nov. 2010, pp. 289-294.

[3] J. Yang and S. Ulukus, "Transmission completion time minimization in an energy harvesting system," in IEEE Proc. Conf. Inf. Sc. Syst., Princeton, NJ, Mar. 2010, pp. 1-6.

[4] M. A. Antepli, E. U. Biyikoglu, and H. Erkal, "Optimal packet scheduling on an energy harvesting broadcast link," IEEE J. Sel. Areas Commun., vol. 29, pp. 1721-1731, Sep. 2011.

[5] V. Sharma, U. Mukherji, V. Joseph, and S. Gupta, "Optimal energy management policies for energy harvesting sensor nodes," IEEE Trans. Wireless Commun., vol. 9, pp. 1326-1336, Apr. 2010.

[6] M. Gatzianas, L. Georgiadis, and L. Tassiulas, "Control of wireless networks with rechargeable batteries," IEEE Trans. Wireless Commun., vol. 9, pp. 581-593, Feb. 2010.

[7] B. Medepally, N. B. Mehta, and C. R. Murthy, "Implications of energy profile and storage on energy harvesting sensor link performance," in Proc. IEEE Glob. Commun. Conf., Honolulu, HI, pp. 1-6.

[8] N. Pappas, J. Jeon, A. Ephremides, and A. Traganitis, "Optimal utilization of a cognitive shared channel with a rechargeable primary source node," J. Commun. Netw., Jul. 2011, submitted for publication.

[9] B. Medepally and N. B. Mehta, "Voluntary energy harvesting relays and selection in cooperative wireless networks," IEEE Trans. Wireless Commun., vol. 9, pp. 3543-3553, Nov. 2010.

[10] H. Li, N. Jaggi, and B. Sikdar, "Cooperative relay scheduling under partial state information in energy harvesting sensor networks," in Proc. IEEE Glob. Commun. Conf., Miami, FL, Dec. 2010, pp. 1-5.

[11] I. Krikidis, B. Rong, and A. Ephremides, "Cooperation for multipleaccess channel via dynamic decode-and-forward," IEEE Trans. Inf. Theory, vol. 57, Dec. 2011, to be published.

[12] A. K. Sadek, K. J. R. Liu, and A. Ephremides, "Cognitive multiple access via cooperation: Protocol design and performance analysis," IEEE Trans. Inf. Theory, vol. 53, pp. 3677-3696, Oct. 2007.

[13] R. Loynes, "The stability of a queue with non-interdependent inter arrival and service times," Proc. Cambridge Philos. Soc., vol. 58, pp. 497-520, 1962. 